\title{
Pc5 waves generated by substorm injection: a case study
}

\author{
N. A. Zolotukhina, P. N. Mager, and D. Yu. Klimushkin \\ Institute of Solar-Terrestrial Physics, P.O. Box 291, 664033 Irkutsk, Russia \\ Received: 15 February 2008 - Revised: 30 April 2008 - Accepted: 12 June 2008 - Published: 28 July 2008
}

\begin{abstract}
We analyzed the spectral-polarized characteristics of Pc5 ULF waves observed on 17 September 2000 after the 03:20:25 UT substorm onset with the satellites GOES 8 and 10 located east and west of the onset location. In the course of the event, the wave polarization changed from mixed (between toroidal and poloidal) to poloidal, and then to mixed again. The hodogram of magnetic field oscillations rotated counterclockwise at GOES 8, and clockwise at GOES 10. It is suggested that the satellites detected the waves generated by the substorm injected clouds of the charged particles drifting in the magnetosphere in the opposite azimuthal directions: GOES 8 (located east of the substorm onset) detected the wave generated by an electron cloud, and GOES 10 (west of the onset) detected the wave generated by a positive ion cloud. This interpretation is confirmed by the energetic particles data recorded by LANL satellites.
\end{abstract}

Keywords. Magnetospheric physics (MHD waves and instabilities) - Space plasma physics (Kinetic and MHD theory)

\section{Introduction}

Poloidal ULF waves are often observed with satellites (Eriksson et al., 2005; Schäfer et al., 2007) and radars (Yeoman et al., 2000). There are some hints that the waves are generated by substorm injected particles drifting in the magnetosphere. This is supported by statistical relations between high- $m$ pulsations and ring current intensifications (Anderson et al., 1991) and observations of events when the waves appeared in some azimuthal location simultaneously with a cloud of substorm injected protons (e.g. Wright et al., 2001). Various kinetic instabilities are often considered, which can generate a ULF wave by means of wave-particle resonances. Among them are drift-bounce instability (Karpman et al., 1977; Mager and Klimushkin, 2005), mirror insta-

Correspondence to: N. A. Zolotukhina

(zolot@iszf.irk.ru) bility (Hasegawa, 1969; Klimushkin and Chen, 2006), drift anisotropy instability (Pokhotelov et al., 1986) and others (see Klimushkin, 2007, for short overview). Still, they cannot be regarded as well established generation mechanisms since, although a particle injection is a transient process, the problem of spatio-temporal evolution of the wave field generated by these instabilities in such nonstationary conditions neither have been solved nor even posed. Other shortcomings of these generation mechanisms are discussed in Mager and Klimushkin (2005); Klimushkin (2007).

Yet another mechanism was suggested by Zolotukhina (1974) and Guglielmi and Zolotukhina (1980): non-resonant generation by moving clouds of substorm injected particles (like Cerenkov emission). Mager and Klimushkin (2007, 2008) developed an analytical theory of the spatio-temporal evolution of the wave field generated by this mechanism in a curved magnetic field (a simple semi-qualitative picture of it is presented in this paper).

The choice between different generation mechanisms is not an easy task, since most data does not uniquely determine between the different theories. This paper reports a case where the relevance of the injection to the waves is very clear. The substorm onset 17 September 2000 took place between the geostationary satellites GOES 8 and 10, and short time later transverse Pc5 waves were observed with both satellites. The observation of the waves west and east makes very probable that the satellites detected the waves generated by clouds of substorm injected ions and electrons. The observed temporal evolution of the wave field is in a reasonably good agreement with the theory of the wave generation by a moving source.

\section{Data: 17 September 2000 substorm associated event}

The substorm happened on 17 September 2000, with the onset at $03: 20: 25 \mathrm{UT}$ at the geographical longitude $\lambda=266^{\circ}$ (Frey et al., 2004). Shortly after the onset, geostationary satellites GOES 8 and 10 registered variations of magnetic field. Since the satellites have the geographical longitudes

Published by Copernicus Publications on behalf of the European Geosciences Union. 


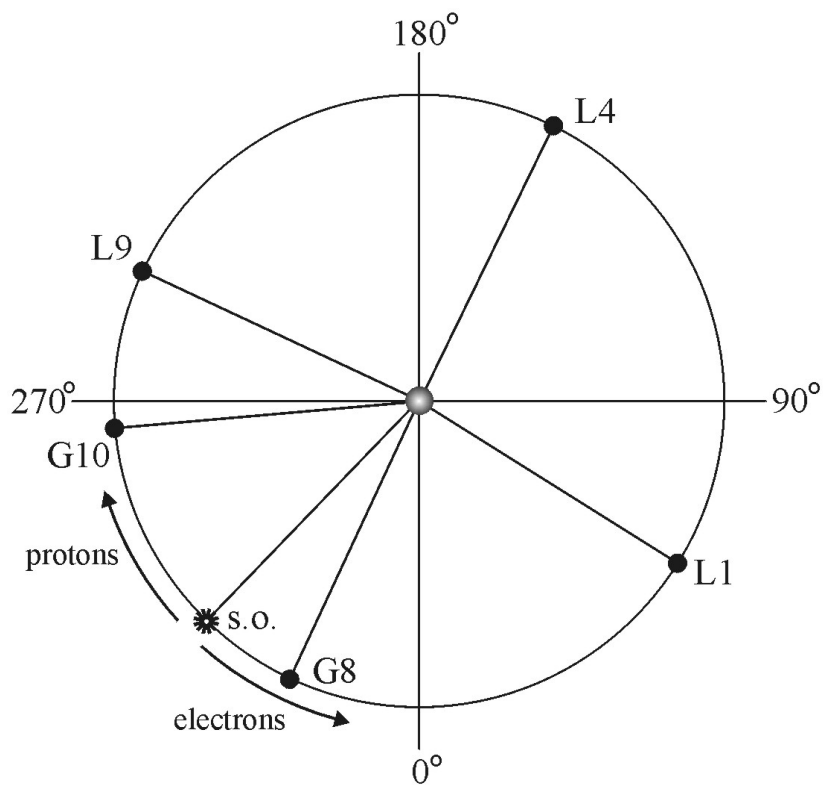

Fig. 1. The position of the substorm onset and the satellites for the moments of the observations of the events.

$\lambda=285^{\circ}$ (GOES 8) and $\lambda=225^{\circ}$ (GOES 10), the onset took place between the satellites: GOES 8 was located about $20^{\circ}$ east of the onset, and GOES 10 was located $40^{\circ}$ west of it (Fig. 1).

The satellites' data, given in the GSE coordinate system (http://cdaweb.gsfc.nasa.gov/), were transformed into the mean-field-aligned coordinate system: the $\mathrm{x}$ - and $\mathrm{y}$ coordinates are directed outward (radial coordinate) and eastward (azimuthal coordinate), and z-coordinate is directed along the averaged magnetic field (defined by taking the 40 min boxcar running averages of the 60-s data (Kim et al., $2002)$. Hence, the transformed $B_{x}(t), B_{y}(t)$, and $B_{z}(t)$ variations approximately describe the poloidal, toroidal and fieldaligned components of magnetic field vector $\boldsymbol{B}$, respectively.

In order to determine a duration of the onset-related magnetic field fluctuations throughout the entire Pc5 period band, the $B_{x}, B_{y}$, and $B_{z}$ components were band-pass filtered in the $T=150-600$ s period range by means of the Marmet (1979) filter. The filtered $B_{x}(t)$ and $B_{y}(t)$ variations are shown in the middle panels of Figs. 2 and 3. One can see that the amplitude of the 150-600-s fluctuations increased noticeably immediately after the onset at GOES 8 and 12-14 min after the onset at GOES 10. Hence, the onset-associated enhancement of the 150-600-s activity lasted almost $80 \mathrm{~min}$, from 03:20 to $04: 40$ UT.

Auto-spectra of unfiltered $B_{x}, B_{y}$, and $B_{z}$ components calculated with the Fourier transform from 80-min time sequences consist of numerous minor and major peaks superimposed on a noise. To find out the proper quasimonochromatic Pc5 waves, we used the technique described

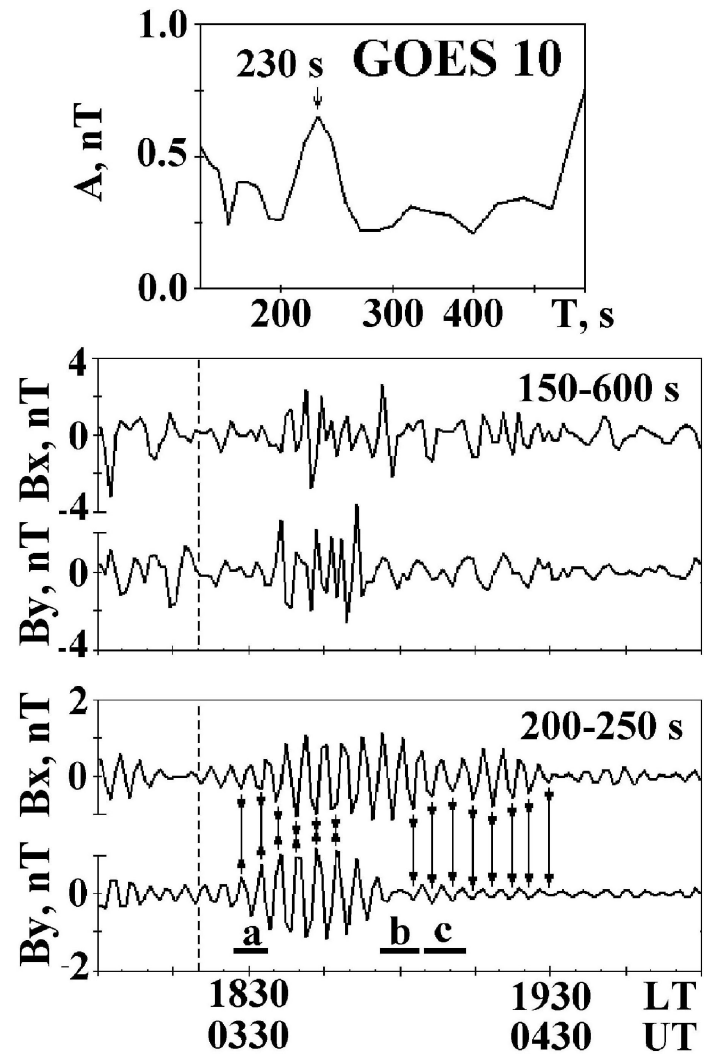

Fig. 2. Features of the 17 September 2000 event in an xy-plane as seen by GOES 10. From the top to the bottom: the spectrum of polarization ellipse major semi-axis, the $B_{x}$ and $B_{y}$ time variations filtered in the entire Pc5 range and in the range near the spectral peak. The substorm onset is marked by a dotted line. Some of the $B_{x}(t)$ and $B_{y}(t)$ maxima/minima are marked by arrows pointing up/down.

in detail by Rankin and Kurtz (1970). Applied to the twodimensional magnetic data series, this technique allows one to determine the coherent, polarized component of pulsations from their two-dimensional spectral power matrix, and calculate parameters of the wave polarization ellipse.

The spectra of the polarization ellipse major semi-axis $A(T)$ were calculated from $B_{x}$ and $B_{y}$ components with the time window mentioned above (see Figs. 2 and 3, top panels). The spectra $A(T)$ show the peaks at 220 s (GOES 8) and $230 \mathrm{~s}$ (GOES 10) which can be considered as the narrowband Pc5. It is significant that there are no spectral maxima at the same periods in $B_{z}$ auto-spectra from GOES 8 and GOES 10. Thus, we believe that in the case under study the Pc5 were the transverse magnetic field oscillations.

Since the spectral analysis reveals only the mean properties of the waves in the specified time interval and gives no information on their time evolution, we extracted a quasimonochromatic Pc5 signal with the 200-250-s Marmet filter, and analyzed it with the hodogram technique. The pulsations 


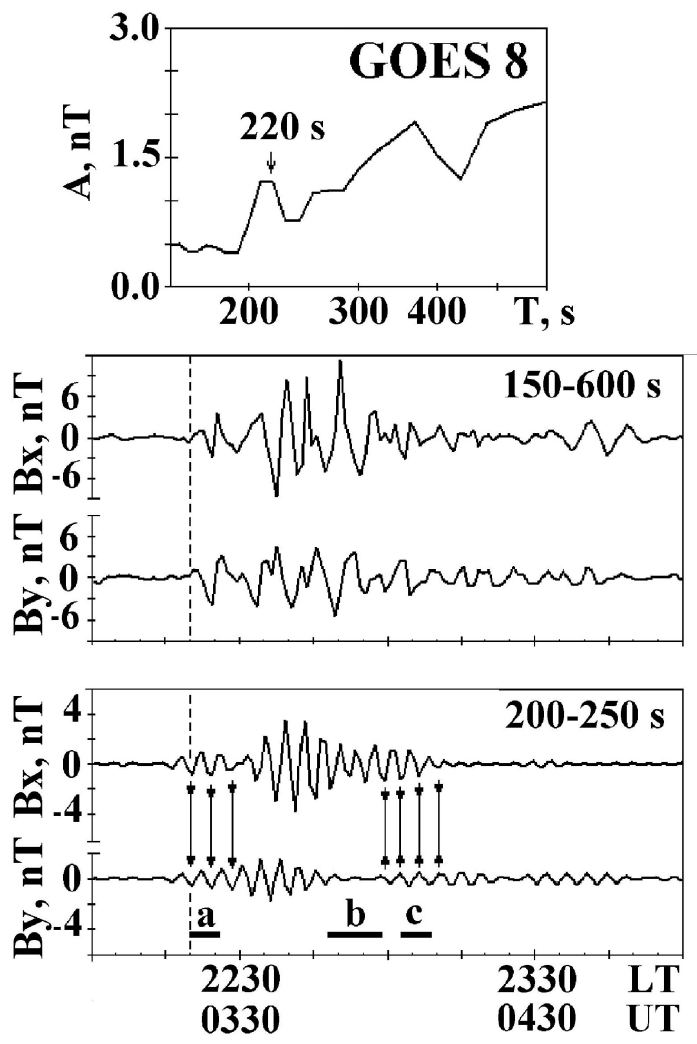

Fig. 3. The same as in the previous figure, but for GOES 8 .

of the 200-250-s filtered $B_{x}$ and $B_{y}$ components are shown in the bottom panels of Figs. 2 and 3. The hodograms of the 200-250 s filtered magnetic field oscillations in the $x y$ plane are depicted in Fig. 4 for time intervals marked in Figs. 2 and 3 by line segments a-c.

From Figs. 2 and 3 and especially Fig. 4 we notice that the direction of the transverse magnetic field oscillations $\left(B_{\perp}\right)$ changes with time. At GOES $8 B_{\perp}$ rotates counterclockwise: $B_{\perp}$ oscillates along the $(+x,+y) \leftrightarrow(-x,-y)$ direction at the beginning, along the $\mathrm{x}$-axis at the midpoint, and along the line $(+x,-y) \leftrightarrow(-x,+y)$ at the end. The sign of $B_{\perp}$ rotation at GOES 10 is opposite to that at GOES 8: it rotates clockwise from the beginning to the end of the wave packet. Accordingly, the polarization of the waves detected by both satellites changes from mixed (between poloidal and toroidal) to poloidal, and then to mixed again.

After GOES 8 and 10 observed the magnetic fluctuations, the LANL satellites registered an increase in the particle fluxes (positions of the satellites are depicted in Fig. 1). In general, an order of the events corresponded to the following pattern: the further from the onset the increase took place, the later the increase occurred.

West of the onset, the increase was in proton flux $J_{p}$ : first it was registered by the LANL 9 satellite located $\Delta \lambda=71^{\circ}$ west of the onset in $\Delta t=18-25 \mathrm{~min}$ after the onset, then by

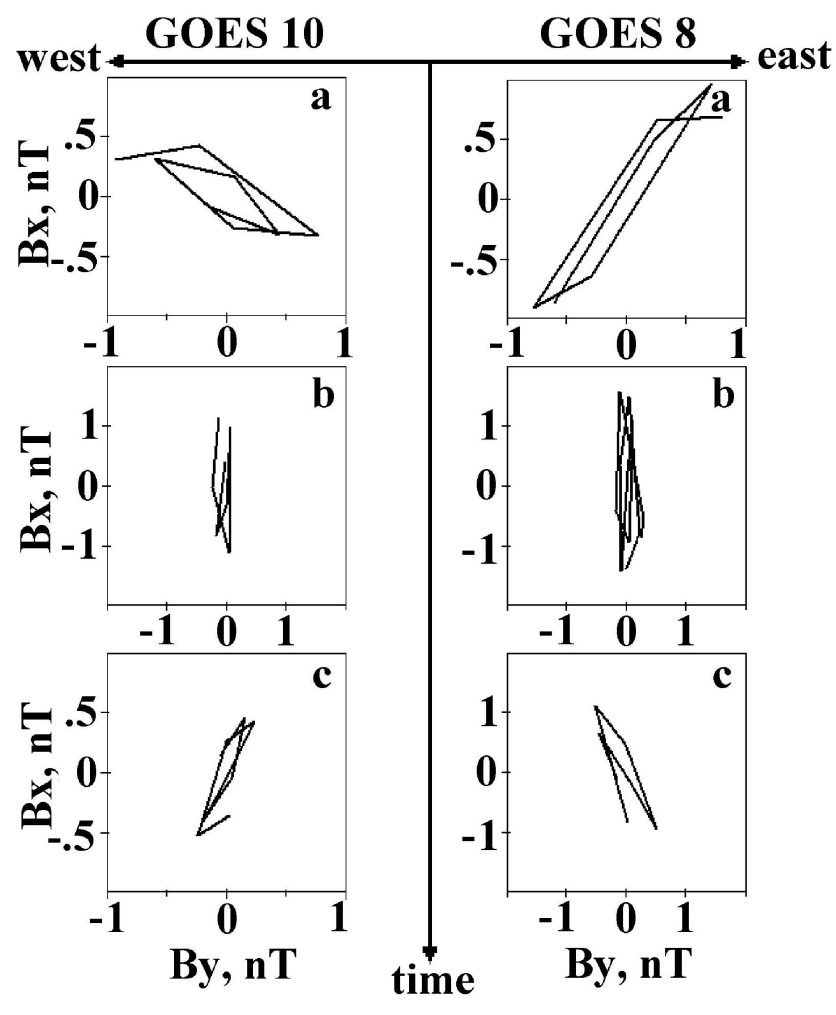

Fig. 4. The hodograms of the waves detected by GOES 10 (left) and GOES 8 (right) in the 200-250 s band.

LANL $4\left(\Delta \lambda=162^{\circ} \mathrm{W}, \Delta t=28\right.$ to $\left.47 \mathrm{~min}\right)$ and even later by LANL $1\left(\Delta \lambda=258^{\circ} \mathrm{W}, \Delta t=40\right.$ to $\left.70 \mathrm{~min}\right)$. In all three cases, the increase was observed in the $50-400 \mathrm{keV}$ channel. With promotion in the west direction, the flux growth rate was damping: $\dot{J}_{p}=14 \mathrm{p} /\left(\mathrm{keV} \mathrm{cm}^{2} \mathrm{sr} \mathrm{s}^{2}\right)$ at LANL 9, $\dot{J}_{p}=7 \mathrm{p} /\left(\mathrm{keV} \mathrm{cm}^{2} \mathrm{sr} \mathrm{s}^{2}\right)$ at LANL $4, \dot{J}_{p}=3 \mathrm{p} /\left(\mathrm{keV} \mathrm{cm}^{2} \mathrm{sr} \mathrm{s}^{2}\right)$ at LANL 1.

These data are naturally interpreted in terms of the proton cloud injected onto the geostationary orbit during the substorm breakup. The cloud was drifting westward and had performed an almost complete turn around the Earth. The damping of flux growth rate $\dot{J}_{p}$ in the course of the drift is a natural consequence of the cloud spreading, as is expected in the case of the injection localized in the azimuthal coordinate. With knowledge of the LANL 9 angular distance (LANL 9 was the nearest satellite to GOES 10) from the point of the substorm onset, $\Delta \lambda=71^{\circ} \mathrm{W}$, and the corresponding time lag $\Delta t=18-25 \mathrm{~min}$, it is possible to calculate the drift velocities of the particles comprising the leading front of the proton cloud: $\omega_{d}=3^{\circ}$ to $4^{\circ}$ per minute. Thus, at the instant of GOES 10 magnetic observations, the proton cloud was somewhere nearby this satellite.

The analogous picture was observed for electron flux $J_{e}:$ it was registered first by LANL $1\left(\Delta \lambda=102^{\circ} \mathrm{E}\right.$, 
$\Delta t=25$ to $50 \mathrm{~min})$ then by LANL $4\left(\Delta \lambda=198^{\circ} \mathrm{E}, \Delta t=44\right.$ to $66 \mathrm{~min})$. The increase was observed in the 50 $225 \mathrm{keV}$ channel, the flux growth rate was damping in the course of time: $\dot{J}_{e}=1100 \mathrm{e} /\left(\mathrm{keV} \mathrm{cm}^{2} \mathrm{sr} \mathrm{s}^{2}\right)$ at LANL 1, $\dot{J}_{e}=80 \mathrm{e} /\left(\mathrm{keV} \mathrm{cm}^{2} \mathrm{sr} \mathrm{s}^{2}\right)$ at LANL 4 . The interpretation is pretty obvious: the substorm injected eastward drifting electron cloud was sequentially observed with the satellites. It should be noted that flux growth rate $\dot{J}_{e}$ at LANL 4 was an order of magnitude less than at LANL 1 , which was located almost two times closer to the point of injection.

\section{Interpretation: high- $m$ Alfvén waves generated by a moving substorm injected cloud of particles}

In the inhomogeneous geomagnetic field, particles of opposite charges drift in opposite directions: negative particles (electrons) drift eastward, and positive particles (ions) drift westward. This immediately provides an interpretation of the double event reported in the previous section: GOES 8 (located east of the substorm onset) detected waves generated by drifting electrons, and GOES 10 (west of the onset) detected waves generated by positive ions. Thus, we have a heuristic model of the event: substorm injected clouds of particles (with opposite charges) directly drive the waves. It means that a cloud produces an external (azimuthal) current $j_{\text {ext }}$ in the Ampère law

$\nabla \times \boldsymbol{B}=\frac{4 \pi}{c} \boldsymbol{j}+\frac{4 \pi}{c} \boldsymbol{j}_{\mathrm{ext}}$,

where $\boldsymbol{B}$ is the wave magnetic field and $\boldsymbol{j}$ is the current associated with the wave. The external current is expressed in terms of the number density $n$ particles in the cloud, the bounce-averaged curvature/gradient drift frequency $\omega_{d}$, and the equatorial distance from the Earth $L$, as

$j_{\mathrm{ext}}=e n \omega_{d} L \delta\left(\varphi-\omega_{d} t\right)$.

The delta-function here shows that the cloud is highly localized in azimuthal coordinate $\varphi$ and arrives at the place with the coordinate $\varphi$ at time instant, defined as $t=\varphi / \omega_{d}$. Here the drift frequency in the inhomogeneous field is expressed through energy $\epsilon$ as

$\omega_{d} \simeq 8 \times 10^{-3} \epsilon L$,

where $\omega_{d}, L$, and $\epsilon$ are measured in degrees per minute, Earth's radii, and keV, respectively (Roederer, 1970).

It should be noted that just after the injection the external current cannot be balanced by the gradient of the plasma pressure. The transition to the steady situation needs some time.

The generation of the Alfvén wave by a cloud of substorm injected particles in a curved magnetic field was studied by Mager and Klimushkin $(2007,2008)$ in terms of the stationary phase method. But the main features of the spatiotemporal structure of the wave field can be elucidated in a rather simple qualitative way.
Let us consider a cloud of particles injected into the axially-symmetric magnetosphere at some time instant $t=0$. This cloud can be considered as an impulse propagating from one location on the azimuthal coordinate to another at drift angular velocity $\omega_{d}(x)$.

Wave excitation by a sudden impulse in the entire magnetosphere is well studied theoretically (e.g. Mann and Wright, 1995; Leonovich and Mazur, 1998). In this case, the azimuthal wave number $m$ may be prescribed arbitrarily. Each field line oscillates with its own eigenfrequency $\omega$, which depends on the radial coordinate $x$ (the coordinate across magnetic shells). Hence, the electric field of the wave is determined by the expression

$E_{x, y}=\left|E_{x, y}\right| e^{-i \omega(x) t+i m \varphi}$,

where $\varphi$ is the azimuthal angle and $y$ denotes the azimuthal coordinate, which can be defined as $y=\varphi L$. In the course of the evolution the wave structure becomes smaller-scale in the radial direction, due to the phase mixing, and an initially poloidally polarized wave transforms into a toroidal one.

For the azimuthally drifting source, the $m$ number is determined by the generation mechanism. In the reference system of the source, the phase just behind the source must be constant, thus the Doppler-shifted wave frequency $\omega^{\star}=\omega-m \omega_{d}$ must be zero. Hence, we obtain the expression for the $m$ number:

$m=\frac{\omega(x)}{\omega_{d}(x)}$.

Note that this value depends on the radial coordinate. Hence, Eq. (4) is replaced by

$E_{x, y}=\Theta\left(\omega_{d} t-\varphi\right)\left|E_{x, y}\right| e^{i \Psi}$.

Here $\Psi$ is the wave phase determined as

$\Psi=-\omega(x) t+\frac{\omega(x)}{\omega_{d}(x)} \varphi$.

The Heaviside step function $\Theta\left(\omega_{d} t-\varphi\right)$ indicates that the wave appears in some azimuthal location simultaneously with the particle cloud arrival in the same spot because the energy of the Alfvén wave almost does not propagate transverse to the field lines: the wave can be viewed as to be carried by the cloud.

Let us describe the main features of the solution obtained. The wave frequency is fully determined by the radial coordinate and does not depend on the nature of the source. So, if two clouds are drifting in opposite azimuthal directions, they must generate waves with the same frequencies determined by the eigenfrequency on this magnetic surface: $\omega=\omega(L)$. It is in agreement with the data described in the previous section, since the waves observed by both satellites have almost the same frequencies. But the azimuthal wave number depends on the particle energy because it is defined through the equality (5) and the drift frequency depends on the particle 
energy according to Eq. (3). So, one can expect different $|m|$ values for the wave generated by electrons and ions. The radial $k_{x}$ and azimuthal $k_{y}$ components of the wave vector are defined through the phase as $k_{x}=\partial \Psi / \partial x$ and $k_{y}=\partial \Psi / \partial y$. The Alfvén wave magnetic field oscillates transverse to the perpendicular wave vector, $\boldsymbol{B}_{\perp} \cdot \boldsymbol{k}_{\perp}=0$, hence the ratio of the radial and azimuthal magnetic field components is

$$
\frac{B_{x}}{B_{y}}=-\frac{k_{y}}{k_{x}} .
$$

Here the azimuthal component is $k_{y}=m / L$. Its sign is determined by the direction of the drift. It points westward (eastward) if the wave is excited by ions (electrons).

Let $t_{0}=\varphi / \omega_{d}$ be a time instant when the source reaches the point with a given azimuthal coordinate $\varphi$. The Heaviside function $\Theta\left(\omega_{d} t-\varphi\right)$ in the Eq. (6) shows that if $t<t_{0}$, the wave field is absent.

The wave vector radial component is determined as

$k_{x}=\Psi^{\prime}=-\left(\frac{\omega}{\omega_{d}}\right)^{\prime}\left(\omega_{d} t-\varphi\right)-\omega t \frac{\omega_{d}^{\prime}}{\omega_{d}}$

(prime means the radial derivative). Its sign depends on the drift velocity profile and varies with space and time. Let us consider the most interesting case when the drift frequency increases with the radial coordinate. Since in most of the magnetosphere the Alfvén eigenfrequency decreases with $L$, $\omega^{\prime}<0$, then the first right-hand side term of the previous equation is positive when $t>t_{0}$ and grows with time, but the second one is always negative. Thus, there is also another special time instant,

$t_{1}=\varphi \frac{\left(\omega / \omega_{d}\right)^{\prime}}{\omega^{\prime}}$.

When $t_{0}<t<t_{1}$, the wave vector radial component is negative, $k_{x}<0$. Both $B_{x}$ and $B_{y}$ components are presented in the wave field, and the mode has a mixed polarization (intermediate between toroidal and poloidal). At the moment $t_{1}$ this value becomes zero, $k_{x}=0$. It is the moment when the mode is purely poloidal for the given location. When $t>t_{1}$, the wave vector radial component becomes positive. In the course of time, $k_{x}$ grows, which means that the mode trasforms from poloidal to toroidal. A reason for this transformation is the phase mixing. Indeed, for $t \gg t_{1}$, the first term in Eq. (7) is much larger than the second one, and $k_{x}$ is determined by the derivative of the first term (as in Eq. 4), that is, it grows with time as $k_{x} \simeq-\omega^{\prime} t$.

The corresponding polarization ellipse of the wave is depicted in Fig. 5. It must be noted that the wave is approximately linearly polarized because in the WKB approximation both radial and azimuthal magnetic field components have the same phase. The most interesting feature of these graphs is the change in the polarization ellipse orientation due to the $k_{x}$ sign change. At the beginning, the ellipse is inclined to the $\mathrm{x}$-axis (mixed wave polarization), then it is directed

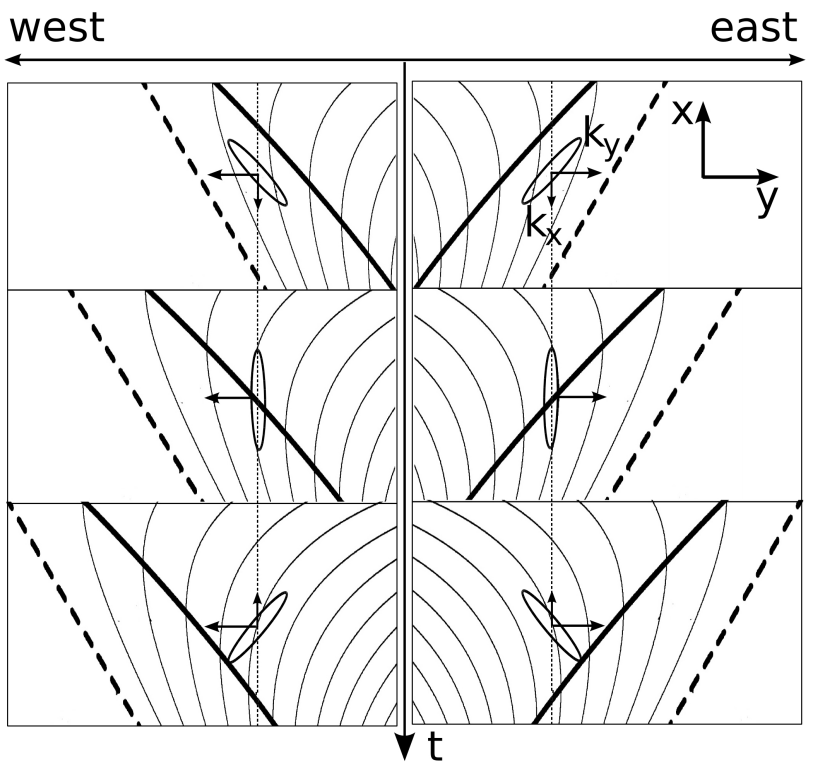

Fig. 5. Polarization ellipses of the wave excited by the moving cloud at different time instants. Dashed lines represent the drifting source. The bold line corresponds to $k_{x}=0$ (purely poloidal polarization of the wave). The right and left parts of the figure correspond to electrons and ions, respectively. Thin lines represent the lines of constant phase.

along this axis (pure poloidal polarization), then it is inclined in the opposite direction (mixed polarization again).

As is seen from comparison of the Figs. 4 and 5, these are the very features observed with the satellites. The hodograms of the wave detected by GOES 8 and 10 rotated counterclockwise and clockwise, respectively, as was expected for the waves generated by the clouds of electrons and ions, the polarization was linear and changes from mixed to poloidal to mixed again. This gives us additional hints for our interpretation.

The theory developed in Mager and Klimushkin (2007, 2008) allows one to evaluate the number density $n$ of the particles responsible for the wave generation. If $B$ is the transverse magnetic field, $c$ is the speed of light, $e$ is the electric charge and $V_{A}$ is the field-line averaged Alfvén speed, then the concentration is

$n \sim \frac{B c}{2 \pi e L V_{A}}$.

Substituting the observed amplitude $B \sim 10^{-5}$ Gauss and $V_{A} \sim 10^{8} \mathrm{~cm} / \mathrm{s}$ into this equation, we obtain $n \sim 10^{-3} \mathrm{~cm}^{-3}$.

It is possible also to evaluate the energy of the particles responsible for the wave generation. The wave activity at GOES 10 (located about $40^{\circ}$ west of the place of the injection) started 12-14 min after the onset. Using Eq. (3), we obtain the energy of ions $\epsilon \sim 60 \mathrm{keV}$, which is quite a reasonable value. 
Let us compare this estimate with the LANL satellites data, presented in the end of the previous section. The drift velocity $\omega_{d}$ from $3^{\circ}$ to $4^{\circ}$ per minute, calculated from the LANL 9 data $\left(L=6.6 R_{E}\right)$, corresponds to proton energy $\epsilon$ from 55 to $75 \mathrm{keV}$, which is quite close to the previous estimate (note that GOES 10 was situated about $30^{\circ}$ east of LANL 9). At LANL 4 location $\left(\Delta \lambda=162^{\circ} \mathrm{W}\right)$, the drift velocity is $\omega_{d} \sim 3.4^{\circ} / \mathrm{min}$ to $5.8^{\circ} / \mathrm{min}$, which corresponds to the energy from $\epsilon \sim 65$ to $110 \mathrm{keV}$, and at LANL 1 location $\left(\Delta \lambda=258^{\circ} \mathrm{W}\right)$ the drift velocity is $\omega_{d} \sim 3.7^{\circ} / \mathrm{min}$ to $6.5^{\circ} / \mathrm{min}$, and the energy is $\epsilon \sim 70$ to $120 \mathrm{keV}$. It is evident that the energy of the protons, forming the cloud leading (western) front, gradually increases in the course of drift, which can be explained by the non-stationarity and azimuthal non-uniformity of the magnetosphere, as well as by the precipitations of the low energy particles into the ionosphere. Nonetheless, the rough coincidence of the estimates obtained from the GOES 10 and LANL 9 (nearest to GOES 10) data shows that it was a substorm injected westward drifting proton cloud, which was responsible for the generation of the waves seen in the GOES 10 data.

The estimates of the drift velocities and particle energies on the leading (east) front of the electron cloud, obtained from the flux data in the 50-225 keV energy band, are $\omega_{d} \sim 2^{\circ} / \mathrm{min}$ to $4 \% \mathrm{~min}, \epsilon \sim 39$ to $77 \mathrm{keV}$ (LANL 1), and $\omega_{d} \sim 3^{\circ} / \mathrm{min}$ to $4.5^{\circ} / \mathrm{min}, \epsilon \sim 57$ to $85 \mathrm{keV}$ (LANL 4). At the upper value of the drift velocity, $\omega_{d} \sim 4^{\circ} / \mathrm{min}$, obtained from the LANL 1 data, the electron cloud must have arrived to the GOES 8 meridian in $5 \mathrm{~min}$ after the injection. Unfortunately, the small angular distance between the place of the injection and the GOES 8 location, together with the finite azimuthal size of the source and rather rough time resolution make it impossible to perform the estimation of the electron energy from the GOES 8 magnetic data and compare it with the LANL 1-4 results.

\section{Conclusion and discussion}

Just after the 17 September 2000 substorm onset a unique configuration occurred: the place of the injection was right between the satellites GOES 8 and 10, and both satellites observed Pc5 waves after the onset. Later, a chain of LANL satellites registered the substorm injected proton cloud drifting westward and the electron cloud drifting eastward. Thus, we obtain the interpretation of the double event: GOES 8 (located east of the substorm onset) detected the waves generated by drifting electrons, and GOES 10 (west of the onset) detected the waves generated by positive ions.

According to the theory, the phase velocity azimuthal component coincides with the drift velocity of the cloud. The wave frequency on a given L-shell does not depend on the nature of the source, being determined by the Alfvén eigenfrequency on this magnetic surface. So, the frequencies of the waves generated by the clouds drifting in opposite azimuthal directions but on the same magnetic shell must be equal. If the drift velocity grows with the radial coordinate, the additional features of the excited wave are expected: (1) approximately linear polarization of the wave, (2) double change of the polarization (mixed $\rightarrow$ poloidal $\rightarrow$ mixed), and (3) change in the orientation of the polarization ellipse. All these features of the waves emitted by a moving cloud of particles were observed.

The estimated energy of the ions responsible for the wave generation is $\sim 60 \mathrm{keV}$. This estimation is confirmed by the energetic particles data recorded by LANL satellites.

An obvious shortcoming of the model is that the particles are assumed monoenergetic. However, we do not expect that it could substantially change the results, since the nonuniform magnetic field acts as a natural filter: the particles with the different energies drift in the magnetosphere with different velocities. The first population which arrives to the point with the given azimuthal coordinate has the highest energy, and, provided that it has sufficient particle density, it must be the one which generates the wave. What is of crucial importance in the context of the present paper, is the growth of the drift velocity with the radial coordinate, because under this condition the particle population is stretched into a spiral, which leads to the double change in the wave polarization. This kind of the particle populations can take place in the magnetosphere under some conditions (Southwood, 1980).

Since the problem of the temporal evolution of the wave field generated by kinetic instabilities mentioned in the Introduction has not been studied, the key features of the event cannot be explained in terms of these theories. Still, the instabilities cannot be completely ruled out. Any realistic treatment of the instabilities on substorm injected particles must include two steps: (i) non-steady current of drifting energetic particles generate the "embryos" of hydromagnetic fluctuations in a way similar the one described in Sect. 3, (ii) a resonant instability provides amplification of narrow-band waves from these "embryo" oscillations (or, if the criteria of the instabilities are not fulfilled, the oscillations are damped due to the interaction with the particles). Thus, even if the instabilities do work, this double event represents the wave on the first initial stage of the process just described.

It should be added that Saka et al. (1992) observed a Pc5 wave event with $m \sim 1$, associated with increased fluxes of energetic electrons in the magnetosphere and interpreted it as a wave generated by electron injection. Finally, we remind the reader of an almost forgotten suggestion by Rostoker and Lam (1978) that some low- $m$ Pc5 waves could be eigenmodes of the magnetosphere-ionosphere current system. This sounds rather vague, but it can be elucidated if we note that charged particle injections represent a part of this current system, thus it is reasonable to suppose that their mechanism expressed in more familiar MHD terms will look very similar to the one considered in the present paper. However, we do not insist on it. 
Acknowledgements. This work is supported by INTAS grant 051000008-7978, RFBR grant 07-05-00185, Program of the presidium of the Russian Academy of Sciences \#16, OFN RAS \#16, and Russian Science Support Foundation (P. N. Mager).

Topical Editor I. A. Daglis thanks M. Volwerk and another anonymous referee for their help in evaluating this paper.

\section{References}

Anderson, B. J., Potemra, T. A., Zanetti, L. J., and Engebretson, M. J.: Statistical correlation between Pc3-5 pulsations and solar wind/IMF parameters and geomagnetic indices, in: Physics of Space Plasmas (1990) SPI Conference Proceedings and Reprint Series, vol. 10, edited by: Chang, T., Crew, G. B., and Jasperse, J. B., Scientific Publishers Inc., Cambridge, Massachusetts, pp. 419-429, 1991.

Eriksson, P. T. I., Blomberg, L. G., Walker, A. D. M., and Glassmeier, K.-H.: Poloidal ULF oscillations in the dayside magnetosphere: a Cluster study, Ann. Geophys., 23, 2679-2686, 2005, http://www.ann-geophys.net/23/2679/2005/.

Frey, H. U., Mende, S. B., Angelopoulos, V., and Donovan, E. F.: Substorm onset observations by IMAGE-FUV, J. Geophys. Res., 109, A10304, doi:10.1029/2004JA010607, 2004.

Guglielmi, A. V. and Zolotukhina, N. A.: Excitation of Alfvén oscillations of the magnetosphere by the asymmetric ring current, Issled. Geomagn. Aeron. i Fiz. Solntsa, 50, 129-137, 1980 (in Russian).

Hasegawa, A.: Drift mirror instability in the magnetosphere, Phys. Pluids. 12, 2642-2650, 1969.

Karpman, V. I., Meerson, B. I., Mikhailovsky, A. B., and Pokhotelov, O. A.: The effects of bounce resonances on wave growth rates in the magnetosphere, Planet. Space. Sci., 25, 573585, 1977.

Kim, K.-H., Cattell, C. A., Lee, D.-H., Takahashi, K., Yumoto, K., Shiokawa, K., Mozer, F. S., and Andre, M.: Magnetospheric responses to sudden and quasiperiodic solar wind variations, J. Geophys. Res., 107(A11), 1406, doi:10.1029/2002JA009342, 2002.

Klimushkin, D. Yu.: How energetic particles construct and destroy poloidal high- $m$ Alfvén waves in the magnetosphere, Planet. Space Sci., 55, 722-730, 2007.

Klimushkin, D. Yu. and Chen, L.: Eigenmode stability analysis of drift-mirror modes in nonuniform plasmas, Ann. Geophys., 24, 2435-2439, 2006,

http://www.ann-geophys.net/24/2435/2006/.

Leonovich, A. S. and Mazur, V. A.: Standing Alfvén waves in an axisymmetric magnetosphere excited by a non-stationary source, Ann. Geophys., 16, 914-920, 1998, http://www.ann-geophys.net/16/914/1998/.
Mager, P. N. and Klimushkin, D. Yu.: Spatial localization and azimuthal wave numbers of Alfvén waves generated by driftbounce resonance in the magnetosphere, Ann. Geophys., 23, 3775-3784, 2005, http://www.ann-geophys.net/23/3775/2005/.

Mager, P. N. and Klimushkin, D. Yu.: Generation of Alfvén waves by a plasma inhomogeneity moving in the Earth's magnetosphere, Plasma Physics Reports, 33, 391-398, 2007.

Mager, P. N. and Klimushkin, D. Yu.: Alfvén ship waves: high$m$ ULF pulsations in the magnetosphere, generated by a moving plasma inhomogeneity, Ann. Geophys., 26, 1653-1663, 2008, http://www.ann-geophys.net/26/1653/2008/.

Mann, I. R. and Wright, A. N.: Finite lifetimes of ideal poloidal Alfvén waves, J. Geophys. Res., 100, 23 677-23 686, 1995.

Marmet, P.: New digital filter for the analysis of experimental data, Rev. Sci. Instr., 50, 79-83, 1979.

Pokhotelov, O. A., Pilipenko, V. A., Nezlina, Yu. M., Woch, J., Kremser, G., Korth, A., and Amata, E.: Excitation of high-beta plasma instabilities at the geostationary orbit: theory and observations, Planet. Space Sci., 34, 695-712, 1986.

Rankin, D. and Kurtz, R.: Statistical study of micropulsation polarizations, J. Geophys. Res., 75, 5444-5458, 1970.

Roederer, J. G.: Dynamics of geomagnetically trapped radiation, Springer-Verlag, New York, 1970.

Rostoker, G. and Lam, H.-L.: A generation mechanism for Pc5 micropulsations in the morning sector, Planet. Space Sci., 26, 493505, 1978.

Saka, O., Iijima, T., Yamagishi, H., Sato, N., and Baker, D. N.: Excitation of Pc 5 pulsations in the morning sector by a local injection of particles in the magnetosphere, J. Geophys. Res., 97, 10 693-10 701, 1992.

Southwood, D. J.: Low frequency pulsation generation by energetic particles, J. Geomagn. Geoelectr., Suppl. II, 32, 75-88, 1980.

Schäfer, S, Glassmeier, K.-H., Eriksson, P. T. I., Pierrard, V., Fornasson, K.-H., and Blomberg, L. G.: Spatial and temporal characteristics of poloidal waves in the terrestrial plasmasphere: A CLUSTER Case Study, Ann. Geophys., 25, 1011-1024, 2007, http://www.ann-geophys.net/25/1011/2007/.

Wright, D. M., Yeoman, T. K., Rae, I. J., Storey, J., Stockton-Chalk, A. B., Roeder, J. L., and Trattner, K. J.: Ground-based and Polar spacecraft observations of a giant $(\mathrm{Pg})$ pulsation and its associated source mechanism, J. Geophys. Res., 106, 10 837-10 852, 2001.

Yeoman, T. K., Wright, D. M., Chapman, P. J., and Stockton-Chalk, A. B.: High-latitude observations of ULF waves with large azimuthal wavenumbers, J. Geophys. Res., 105, 5453-5462, 2000.

Zolotukhina, N. A.: On excitation of Alfvén waves in the magnetosphere by a moving source, Issled. Geomagn. Aeron. i Fiz. Solntsa, 34, 20-23, 1974 (in Russian). 